\title{
Avaliação da influência dos sintomas clínicos na qualidade de vida de indivíduos com Síndrome de Williams-Beuren
}

\author{
Luísa de Mattos Graziania , Andrea Parolin Jackowski ${ }^{a}$, Rosana Aparecida Salvador Rossit ${ }^{\mathrm{b}}$, \\ Carolina Grego Del Cole ${ }^{\mathrm{a}}$
}

\author{
aDepartamento de Psiquiatria, Universidade Federal de São Paulo - UNIFESP, São Paulo, SP, Brasil \\ bDepartamento de Saúde, Educação e Sociedade, Universidade Federal de São Paulo - UNIFESP, \\ São Paulo, SP, Brasil.
}

\begin{abstract}
Resumo: Introdução: A Síndrome de Williams-Beuren (SWB) é resultado da deleção do cromossomo 7q11.23. A presença de transtornos psiquiátricos, tais como Transtorno de Ansiedade Generalizada, Déficit de Atenção e Hiperatividade, entre outros, já foram descritos nesta síndrome. Objetivo: Este estudo teve como objetivo identificar possíveis sintomas clínicos nos indivíduos com SWB e as possíveis consequências na qualidade de vida. Método: O grupo amostral com SWB constituiu-se por 20 indivíduos com idade entre 11 e 16 anos e 22 indivíduos com desenvolvimento típico (DT). Os instrumentos utilizados foram: Critério de Classificação Econômica Brasil para estimar o poder de compra das famílias urbanas; o Questionário de Capacidades e Dificuldades (SDQ), que possibilita uma medida útil em psicopatologia aplicada a crianças e jovens com 4 a 17 anos de idade; e o Questionário de Avaliação de Qualidade de Vida em Crianças e Adolescentes (AUQEI), que tem como objetivo avaliar a sensação de bem-estar mediante a opinião da própria criança e adolescente. Resultados: Os resultados sugerem que os adolescentes com SWB possuem uma boa qualidade de vida, apesar de apresentarem maiores possibilidades de sintomas psiquiátricos. Estes resultados são válidos, já que a qualidade de vida é mensurada a partir da subjetividade do indivíduo avaliado. Conclusão: Chega-se à hipótese de que os adolescentes com SWB podem apresentar uma distorção da realidade para o fator positivo/otimista, possivelmente baseada em algumas características da própria síndrome, tais como: são sempre alegres e sorridentes, fatores associados à deficiência intelectual.
\end{abstract}

Palavras-chave: Síndrome de Williams, Sintomas Clínicos, Qualidade de Vida.

\section{Evaluation of the influence of clinical symptoms on the quality of life in individuals with Williams-Beuren Syndrome}

\begin{abstract}
Introduction: Williams-Beuren Syndrome (WBS) results from a deletion in the chromosome 7q11.23. Psychiatric symptoms, such as Generalized Anxiety Disorder (GAD), Attention Deficit Hyperactivity Disorder (ADHD), and others, have been described in this syndrome. Objective: The main purpose of this study was to identify related clinical symptoms in individuals with WBS and the impact of this disorder in their quality of life. Method: The study sample included 42 individuals aged 11 to 16 years divided into two groups: a study group composed of 20 individuals with WBS and a control group comprising 22 individuals with typical development. The following instruments were used in this evaluation: Brazilian Criterion of Economic Classification (CCEB), to estimate the purchasing power of urban households; Strengths \& Difficulties Questionnaire (SDQ), to provide a useful measure of psychopathology applied to children and youth aged 4-17 years; and Quality of Life Evaluation Scale (AUQEI), to assess the feeling of well-being according to the opinion of the child and adolescent. Results:
\end{abstract}

Autor para correspondência: Carolina Grego Del Cole, Laboratório de Neurociências Clínicas, Departamento de Psiquiatria, Universidade Federal de São Paulo, Rua Pedro de Toledo, 669, $3^{\circ}$ andar fundos, Edifício de Pesquisas II, Vila Clementino, CEP 04039-032, São Paulo, SP, Brasil, e-mail: carolinadelcole@gmail.com

Recebido em Out. 14, 2015; $1^{\text {a }}$ Revisão em Jan. 26, 2016; 2 ${ }^{a}$ Revisão em Maio 19, 2016; Aceito em Jul. 6, 2016. 
The results suggest that adolescents with WBS have a good quality of life despite presenting greater possibilities of psychiatric symptoms. These results are valid considering that quality of life is measured from the opinion of the assessed individual. Conclusion: We hypothesized that adolescents with WBS can present a distortion of reality regarding the positive/optimistic factor, possibly based on some characteristics of the syndrome such as being always cheerful and smiling, which are factors associated with intellectual disability.

Keywords: Williams-Beuren Syndrome, Clinical Symptoms, Quality of Life.

\section{Introdução}

A Síndrome de Williams-Beuren (SWB) foi descrita pela primeira vez por Williams, Barratt-Boyes e Lowe (1961) e Beuren, Apitz e Harmjanz (1962) que observaram características faciais típicas associadas à estenose supravalvular aórtica, hipercalcemia infantil e deficiência intelectual em pacientes não aparentados. É um distúrbio relativamente raro do desenvolvimento neurológico, resultado de uma deleção hemizigótica de 25-30 genes no cromossomo 7q11.23 (EWART et al., 1993; KORENBERG, 1993; HILLIER et al., 2003). A SWB ocorre em cerca de 1 em cada 8.000 nascidos vivos (MARTENS; WILSON; REUTENS, 2008).

Esta síndrome apresenta maiores potencialidades em habilidades de linguagem contrastada com o severo déficit em habilidade visuoespacial, desta forma, frequentemente é descrita por um perfil de "picos e vales". São considerados altamente sociáveis, sorridentes e com um alto nível de desenvolvimento verbal. Frequentemente apresentam deficiência intelectual leve a moderada e apresentam dificuldades em: raciocínio espacial, solucionar problemas e motricidade fina (NUNES, 2010). Além do mais, exibem um forte impulso para se engajarem socialmente, com aumento da propensão em abordar estranhos (JARVINEN-PASLEY et al., 2008; JAVINEN; BELLUGI, 2013; HAAS; REISS, 2012).

A literatura também descreve os indivíduos com SWB como mais ansiosos, distraídos, hiperativos e propensos em apresentar dificuldades de relacionamento com colegas de mesma idade cronológica, (crianças da mesma faixa etária) ou aqueles com níveis semelhantes de déficit cognitivo (DYKENS, 2003; EINFELD; TONGE; FLORIO, 1997; EINFELD; TONGE; REES, 2001; GREER et al., 1997).

Mervis et al. (2015) avaliaram 79 indivíduos com SWB entre 18 meses e 17 anos de idade e 12 adultos. Os resultados indicaram variaçóes muito amplas em relação às avaliaçôes padronizadas de inteligência, vocabulário e desempenho acadêmico. Os níveis de adaptação variam de deficiência grave a média, assim como, o comportamento disruptivo foi classificado desde a extrema dificuldade à sua ausência. Mais da metade dos participantes foi diagnosticada com transtorno de ansiedade, além disso, um quarto das crianças foi diagnosticada com transtorno desafiador opositivo ou transtornos do comportamento disruptivo - sem outra especificação, e um terço das crianças rastreou positivamente para o transtorno do espectro autista.

Um estudo transversal, analisou o perfil do comportamento adaptativo em 100 adolescentes e adultos com SWB e usou a escala Independent Revised - Comportamento (SIB-R). Os achados indicaram um déficit significativo relativo às habilidades motoras e habilidades de vida em comunidade, em comparação ao desempenho nas interações sociais, habilidades de comunicaçáo e habilidades de vida pessoal (FU, 2015). Malone e Stoneman (1990) e Beadle-Brown et al. (2000) destacam que o desenvolvimento da cogniçáo está associado com o desenvolvimento do comportamento adaptativo $\mathrm{e}$, consequentemente, influencia a qualidade de vida. A terapia ocupacional atua na vertente dos transtornos de saúde mental comuns na infância e na adolescência e visa melhorar a saúde, promover a autonomia e inclusão social (FLEITLICH-BILYK; GOODMAN, 2004).

Um estudo brasileiro (DEL COLE et al., 2013) analisou o desempenho ocupacional em relaçáo às Atividades Básicas de Vida Diária (ABVD) e Atividades Instrumentais de Vida Diária (AIVD) em 10 adolescentes e jovens adultos com SWB e identificou que nove entre dez indivíduos apresentam dependência grave alusiva aos cuidados familiares em ABDV e AIVD. Os principais problemas comportamentais relatados pelos pais envolvem: a falta de atenção, alteração no contato social e excesso de ansiedade. Além disso, este estudo constatou também, dificuldades em interações e relacionamentos pessoais, empobrecimento significativo do repertório social e de papéis ocupacionais, em relação ao contexto social/ambiental em que se encontravam.

Estudos apontam que a qualidade de vida consiste em possessão dos recursos necessários para a satisfação de necessidades e desejos individuais, participação em atividades que permitem o desenvolvimento pessoal, a autorrealização e uma comparação satisfatória 
entre si mesmo e os outros (DANTAS, 1997; SHIN; JOHNSON, 1978; HINDS, 1990). Para crianças e adolescentes, a perspectiva da qualidade de vida também está associada à distância entre seus desejos e a realidade momentânea (CALMAN, 1984). Pesquisas em saúde mental centram-se em questôes de que a baixa qualidade de vida pode ser consequência da disfunção, incapacidade e deficiência (JANSEN et al., 2011).

A partir da perspectiva de que o déficit cognitivo, deficiência física e sintomas clínicos possam afetar a qualidade de vida, a hipótese é de que a percepção dos adolescentes com SWB sobre sua qualidade de vida seja inferior em relação à dos adolescentes de desenvolvimento típico (DT). Assim, o principal objetivo deste estudo foi avaliar se a qualidade de vida de adolescentes com SWB é deficitária em relação a adolescentes com DT e se a presença de sintomas clínicos influencia negativamente a qualidade de vida deles.

\section{Método}

\subsection{Grupo amostral}

O grupo amostral com SWB foi composto por 20 adolescentes e os critérios de inclusão foram: ter idade cronológica entre 11 e 16 anos; apresentar o diagnóstico de SWB confirmado geneticamente por análise clínica e exame de citogenética molecular (Hibridização in situ por Fluorescência - FISH); residir no Estado de São Paulo e ser cadastrado na Associação Brasileira de Síndrome de Williams (ABSW).

O grupo de DT foi constituído por 22 adolescentes que eram parentes ou amigos do grupo com SWB. Os critérios de inclusão estabelecidos foram: residir no Estado de Sáo Paulo e apresentar pareamento com o grupo SWB nos requisitos idade, gênero e classificação econômica.

\subsection{Instrumentos de avaliação}

\subsubsection{Critério de Classificação Econômica Brasil (2011)}

O questionário utilizado foi desenvolvido pela Associação Brasileira de Empresas em Pesquisa (ASSOCIAÇÃO..., 2011), na qual o critério de Classificação Econômica Brasil enfatiza a função de estimar o poder de compra das famílias urbanas e a divisão de mercado é definida exclusivamente por classes econômicas. Foi construído por meio da utilização de técnicas estatísticas que sempre se baseiam em coletivos, de forma que a probabilidade de classificação correta é alta e a probabilidade de erro, baixa. Assim, os casos incorretamente classificados serão pouco numerosos de modo a náo distorcer significativamente os resultados da investigação.

\subsubsection{Questionário de Capacidades e Dificuldades (SDQ)}

O SDQ é um questionário breve de rastreamento de possíveis sintomas psiquiátricos em crianças e adolescentes entre 4 e 17 anos de idade. A versão selecionada para este estudo foi P4-17 - SDQ e impacto suplementar para pais de crianças ou adolescentes. Os 25 itens do SDQ são divididos entre cinco perfis de diagnóstico: sintomas emocionais (5 itens); problemas de conduta (5 itens); hiperatividade/falta de atenção (5 itens); problema de relacionamento com colegas (5 itens); comportamento pró-social (5 itens).

A pontuação geral de dificuldades é gerada pela soma dos resultados de todas as escalas, exceto a escala de comportamento pró-social. O resultado pode variar de 0 a 40 pontos e possíveis sintomas clínicos estáo presentes se ao menos 12 dos 20 pontos forem identificados como característica do sujeito avaliado. O questionário SDQ aponta índices positivos de validade e fidedignidade em 21 países, incluindo o Brasil, caracterizando seu alcance transcultural e sua aplicabilidade na área de saúde mental infantojuvenil (SAUR; LOUREIRO, 2012).

\subsubsection{Questionário de Avaliação de Qualidade de Vida em Crianças e Adolescentes (AUQEI)}

A versão validada da "Escala de Qualidade de Vida da Criança" (do francês Autoquestionnaire Qualité de Vie Enfant Imagé - AUQEI), desenvolvida por Manificat e Dazord (1997), é um instrumento que visa avaliar a sensação subjetiva de bem-estar do indivíduo e parte da premissa de que o indivíduo em desenvolvimento é capaz de se expressar quanto à sua subjetividade. A escala AUQEI é composta por 26 questôes que exploram relaçôes familiares, sociais, atividades, saúde, funções corporais e separação.

Trata-se de uma autoavaliação que utiliza o suporte de imagens, em que o próprio adolescente responde cada questão através do auxílio de faces que exprimem diferentes estados emocionais (Figura 1). $\mathrm{O}$ adolescente é instruído a assinalar, sem tempo definido, a resposta que mais corresponde ao seu sentimento, em relação ao domínio proposto. Porém, 


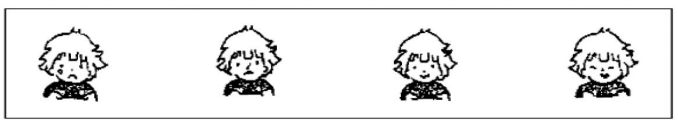

Figura 1. Faces do Questionário de Avaliação de Qualidade de Vida em Crianças e Adolescentes (AUQEI). Nota: Sentimento das faces iniciando da esquerda para a direita: muito triste, triste, feliz e muito feliz.

é solicitado primeiramente, que o adolescente relate uma experiência própria vivida perante cada uma das alternativas, isso permite que ele compreenda melhor as situaçóes, ao apresentar suas próprias experiências. A escala permite obter um perfil de satisfação do indivíduo diante de diferentes situações (ASSUMPÇÃO et al., 2000).

Este instrumento apresenta uma etapa anterior à aplicação das questôes, a fim de assegurar que a criança ou o adolescente expresse seus sentimentos a cada uma das opçóes de respostas. Para a resposta que representa "muito feliz", a criança é induzida a lembrar de alguma situação prévia em que ela teve tal sentimento, a fim de melhor compreender as opçóes de resposta (ASSUMPÇÃO et al., 2000). Além disso, como este instrumento apresenta imagens pouco claras sobre as diferenças entre elas, foi necessário que, durante o processo de avaliaçáo, o pesquisador, por cautela, repetisse em todas as questôes o que o adolescente sentia, ao observar a imagem apontada e correspondente; tal como ao ser questionado: "Quando seus pais falam de você, como você se sente?", "Muito feliz, feliz, triste ou muito triste" (o pesquisador aponta a imagem correspondente).

O AUQEI mostrou propriedades psicométricas satisfatórias e facilidade de utilização. Para validar esse instrumento para a Língua Portuguesa, avaliaram-se 353 escolares de quatro a 12 anos da cidade de Sáo Paulo, SP; foi estabelecido para a população geral um ponto de corte de 48; e considerou-se a qualidade de vida insatisfatória abaixo desse escore. $\mathrm{O}$ estudo apresentou validade interna, com coeficiente de Cronbach de 0,71 e indicou confiabilidade adequada do instrumento (ASSUMPÇÃO et al., 2000).

\subsubsection{Wechsler Intelligence Scale for Children-Third Edition (WISC-III)}

O questionário WISC-III é um instrumento que avalia o desenvolvimento de algumas funçóes cognitivas de crianças e adolescentes com idade entre 6 e 16 anos (WECHSLER, 1991). O Quociente de inteligência (QI) estimado foi calculado por meio da utilização dos subtestes Cubos e Vocabulário. Estes subtestes foram escolhidos por apresentarem características psicométricas que se correlacionam com o QI total (MELLO et al., 2011). As análises mostraram que os itens adaptados apresentaram características psicométricas adequadas que possibilitam a utilizaçáo do instrumento como meio confiável de diagnóstico (FIGUEIREDO et al., 2008).

\subsubsection{Vineland Adaptive Behavior Scale (VABS)}

A escala VABS mensura o comportamento adaptativo e este é um importante dado para auxiliar o diagnóstico de deficiência intelectual (DSM-5). Esta escala avalia a capacidade de o indivíduo lidar com as mudanças ambientais, se aprende a realizar as atividades cotidianas, e se demonstra independência ao desenvolvê-las (SPARROW et al., 1984). A escala se baseia em uma entrevista estruturada com o principal cuidador. $\mathrm{O}$ tempo de avaliação foi de aproximadamente 25 a 90 minutos. VABS é organizada em uma estrutura com três domínios principais: comunicação, atividades de vida diária e socialização. Os resultados brutos obtidos a partir dos domínios foram ponderados para se ajustarem de acordo com a idade cronológica, de acordo com o manual do VABS, e normalizada para obter uma métrica comum (SPARROW et al., 1984). Os dados psicométricos da escala VABS são confiáveis e estes foram descritos em seu manual por meio de evidências de construção, conteúdo e validade de critério (SPARROW et al., 1984).

\subsection{Procedimento}

Os dados foram coletados durante os anos de 2011 e 2012 no Departamento de Psiquiatria da Universidade Federal de São Paulo (UNIFESP), em ambientes reservados e em eventos realizados pela ABSW na cidade de São Paulo. Realizou-se um encontro com a família e o adolescente para a coleta dos dados e o contato inicial foi realizado da seguinte maneira: uma parte foi por telefone e outra, pessoalmente durante os eventos da ABSW.

Depois dos esclarecimentos, compreensão e concordância em relação ao propósito deste estudo e dos procedimentos de avaliação que seriam aplicados, os pais autorizaram a participação dos filhos, assinaram o Termo de Consentimento Livre e Esclarecido e responderam, junto a um aplicador treinado, os seguintes instrumentos: Critério de Classificação Econômica Brasil, SDQ e a escala VABS. 
De forma análoga, primeiramente os adolescentes foram esclarecidos sobre as avaliaçóes e, após a concordância em participar, assinaram o Termo de Assentimento. Em seguida, responderam, junto a um aplicador treinado, o questionário AUQEI e o WISC-III.

O tempo de duração de aplicação do Critério de Classificação Econômica Brasil, SDQ e VABS foi de 1 hora e a aplicação dos questionários AUQEI e WISC-III durou cerca de 40 minutos.

\subsection{Análise dos dados}

Para comparar os grupos em relação a cada uma das variáveis de interesse, empregou-se o teste $t$ de Student para amostras independentes, utilizando-se do software R.

A análise descritiva dos dados apresentados incluiu média e desvio padrão. Para todos os procedimentos estatísticos, o intervalo de confiança foi de $95 \%$ $(\mathrm{p}<0.05)$.

\section{Resultados}

\subsection{Caracterização da amostra - dados sociodemográficos}

Não houve diferença estatística entre os grupos SWB e DT em relação ao gênero ( $>0.287$ ), idade ( $p>0.892)$ e classe social $(p>0.887)$. Entretanto, como era esperado, houve diferença estatisticamente significativa para o QI estimado, para todos os domínios do comportamento adaptativo e o total da escala VABS. Assim, o grupo DT apresentou melhor desempenho em relaçáo ao grupo SWB no QI estimado $(\mathrm{p}<0.001)$ e em todos os domínios da escala VABS $(\mathrm{p}<0.001)$.

No entanto, o tamanho de efeito entre as médias de desempenho do comportamento adaptativo entre os grupos DT e o grupo com SWB foi classificado como efeito de tamanho médio para o domínio de socialização e classificado como efeito de tamanho grande para os domínios de comunicação, atividades de vida diária e comportamento adaptativo total. Estes dados estáo descritos na Tabelas 1 e 2 .

\subsection{Qualidade de vida e dados clínicos}

Não houve diferença estatística entre o grupo com SWB (média $=52.50$ e DP $=8.19$ ) e grupo $\mathrm{DT}$ (média $=51.41$ e DP = 5,16; p >0.613) no que se refere à qualidade de vida como era esperado, sendo que o grupo com SWB obteve um ponto acima da média em relação ao grupo DT (Figura 2).

O grupo com SWB (média = 19,29 e DP = 4,12) apresentou maiores possibilidades de sintomas clínicos quando comparado ao grupo DT (média $=8,05 \mathrm{e}$ $\mathrm{DP}=6,15 ; \mathrm{p}<0.001)$. Os resultados relativos aos subdomínios da escala SDQ foram: os adolescentes com SWB obtiveram uma pontuação inferior à do grupo DT no subdomínio do "comportamento pró-social” ( $\mathrm{p}<0.002)$ (Figura 3). Ademais, como era esperado, o grupo com SWB apresentou maiores possibilidades para hiperatividade $(\mathrm{p}<0.001)$ (Figura 4$)$, problemas com colegas $(\mathrm{p}<0.001)$ (Figura 5$)$, sintomas emocionais $(\mathrm{p}<0.001)$ (Figura 6), e problemas de conduta $(\mathrm{p}<0.05)$ (Figura 7$)$.

Tabela 1. Tabela com dados sociodemográficos.

\begin{tabular}{|c|c|c|c|c|c|c|c|c|c|c|}
\hline & \multicolumn{3}{|c|}{ Genêro } & \multicolumn{2}{|c|}{ Idade } & \multicolumn{3}{|c|}{ Classificação Econômica } & \multicolumn{2}{|c|}{ WISC (QI) } \\
\hline & $\begin{array}{c}\text { Fem. } \\
\text { N }(\%)\end{array}$ & $\begin{array}{l}\text { Masc. } \\
\text { N (\%) }\end{array}$ & $\begin{array}{l}\text { Valor } \\
\text { de P }\end{array}$ & $\begin{array}{l}\text { Média } \\
\text { (SD) }\end{array}$ & $\begin{array}{l}\text { Valor } \\
\text { de P }\end{array}$ & A/B & $C / D$ & $\begin{array}{l}\text { Valor } \\
\text { de P }\end{array}$ & Média (SD) & $\begin{array}{l}\text { Valor } \\
\text { de P }\end{array}$ \\
\hline DT & $9(41)$ & $13(59)$ & - & $13,6(1,8)$ & - & $12(54,5)$ & $10(45,5)$ & - & $105,62(16,77)$ & - \\
\hline SWB & $11(55)$ & $9(45)$ & 0,287 & $13,7(1,8)$ & 0,892 & $11(52,4)$ & $9(47,6)$ & 0,887 & $59,39(9,07)$ & $<, 001 * *$ \\
\hline
\end{tabular}

Nota: Desenvolvimento típico $=$ DT; Síndrome de Williams-Beuren $=$ SWB. Feminino $=$ Fem.; Masculino $=$ Masc. Classes sociais A/B e Classes sociais C/D. **= estaticamente significante.

Tabela 2. Tamanho do efeito da média do comportamento adaptativo nos grupos DT e SWB.

\begin{tabular}{|c|c|c|c|}
\hline & 1 & 2 & HC vs. WBS \\
\hline & DT & SWB & $\begin{array}{l}\text { Cohen's d/ } \\
\text { effect size r }\end{array}$ \\
\hline & Mean (SD) & Mean (SD) & $(p)$ \\
\hline Socialização & $112,45(17,40)$ & $60,30(26,09)$ & $2,35 / 0,76^{*}(p<, 001)$ \\
\hline Comunicação & $103,66(13,57)$ & $40,82(18,95)$ & $3,82 / 0,88^{* *}(p<, 001)$ \\
\hline Atividades de Vida Diária & $103,33(18,89)$ & $33,91(15,57)$ & $4,06 / 0,89 * *(p<, 001)$ \\
\hline Comportamento Adaptativo Total & $108,62(16,42)$ & $41,39(15,39)$ & $3,60 / 0,87 * *(p<, 001)$ \\
\hline
\end{tabular}

Nota: Desenvolvimento típico = DT; Síndrome de Williams-Beuren = SWB; socialização, comunicação e atividades de vida diária, e comportamento adaptativo total da Escala Vineland. * efeito de tamanho médio; **efeito de tamanho grande. 


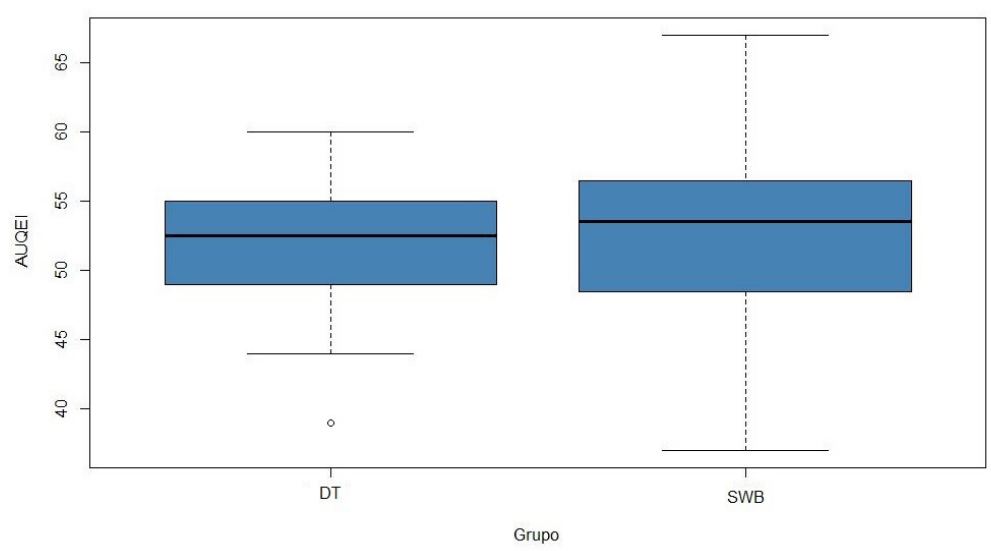

Figura 2. Distribuição da variável 'AUQEI' nos grupos com SWB e DT. Nota: Não houve diferença estatística no que se refere à qualidade de vida entre o grupo SWB e o grupo DT.

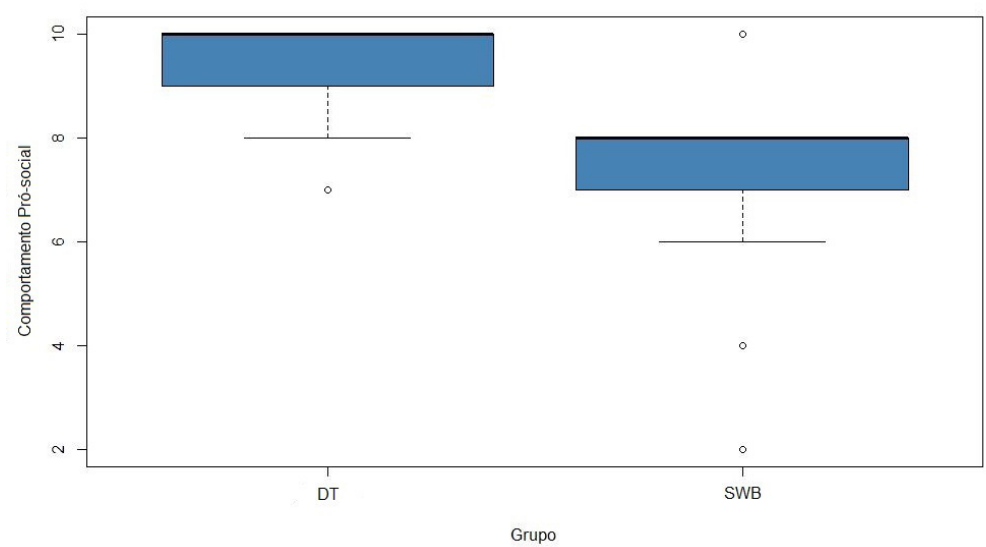

Figura 3. Distribuição do subdomínio 'comportamento pró-social' nos grupos com SWB e DT. Nota: Indivíduos com SWB tiveram uma pontuação inferior à do grupo DT na variável "comportamento prósocial".

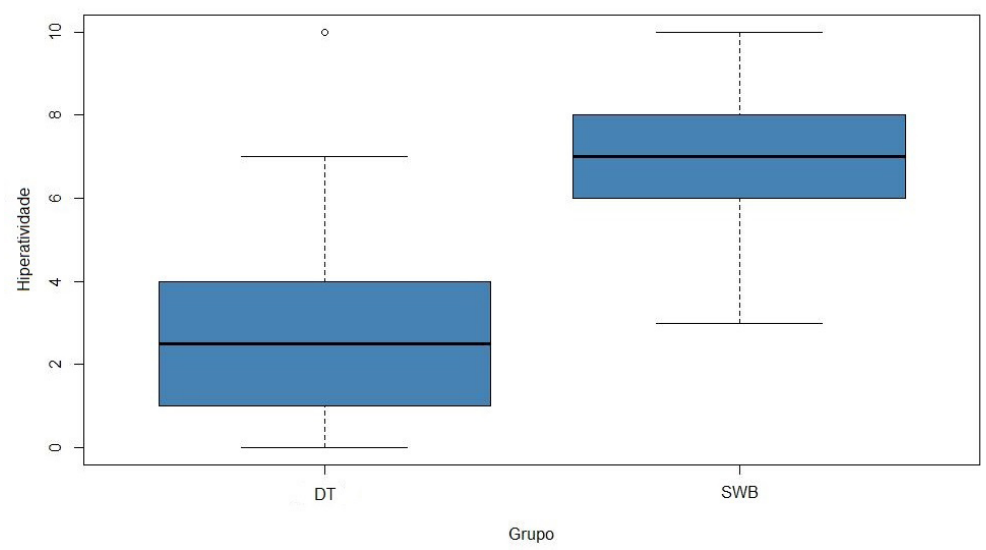

Figura 4. Distribuição do subdomínio 'hiperatividade’ nos grupos com SWB e DT. Nota: Indivíduos com SWB tiveram uma pontuação superior à do grupo DT na variável "hiperatividade". 


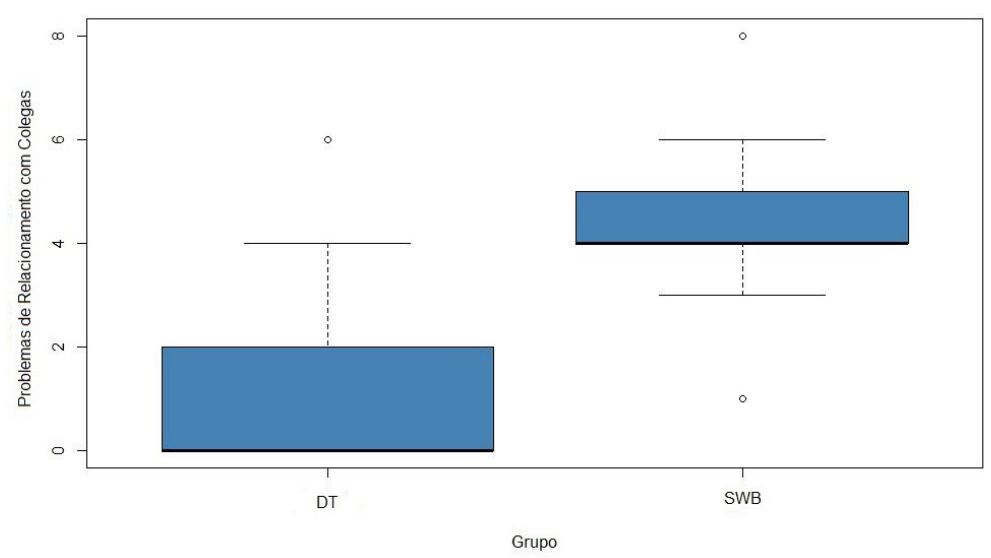

Figura 5. Distribuição do subdomínio 'problemas de relacionamento com colegas' nos grupos com SWB e DT. Nota: Indivíduos com SWB tiveram uma pontuação superior à do grupo DT na variável "problemas de relacionamento com colegas".

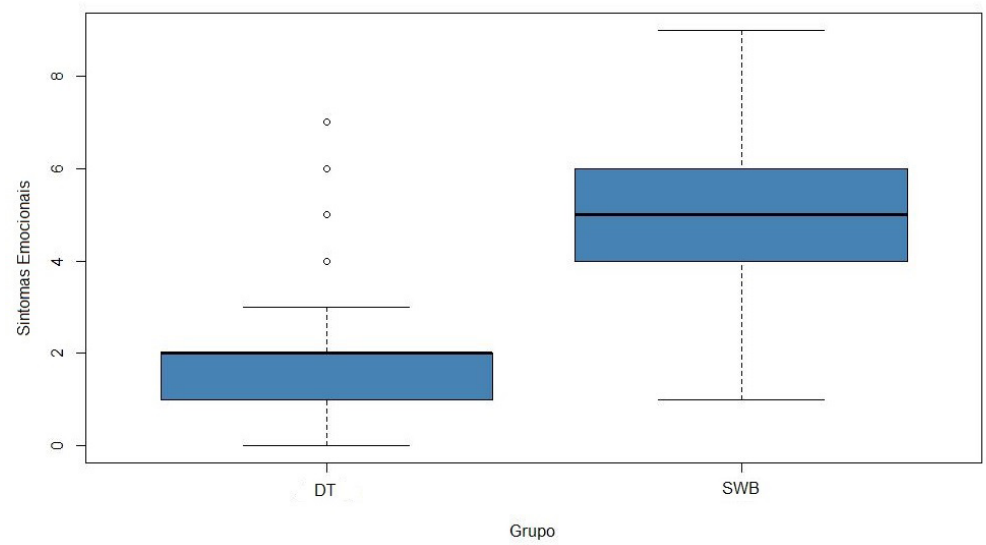

Figura 6. Distribuição do subdomínio ‘sintomas emocionais' nos grupos com SWB e DT. Nota: Indivíduos com SWB tiveram uma pontuação superior à do grupo DT na variável "sintomas emocionais".

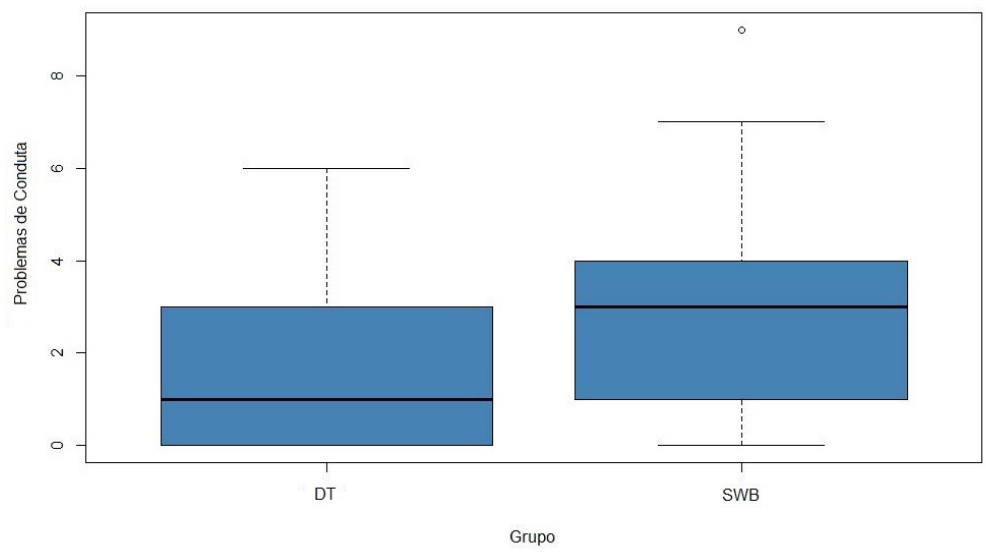

Figura 7. Distribuição do subdomínio 'problemas de conduta' nos grupos com SWB e DT. Nota: Indivíduos com SWB tiveram uma pontuação superior à do grupo DT na variável "problemas de conduta". 


\section{Discussão}

O presente estudo mostrou que náo houve diferença na qualidade de vida entre o grupo SWB e o grupo DT. Todavia, embora os possíveis sintomas clínicos sejam evidentes no grupo com SWB, tais sintomas não interferiram na qualidade de vida destes adolescentes.

Os achados relativos aos sintomas clínicos corroboram com a literatura, pois relatam que os indivíduos com SWB são mais ansiosos, distraídos, hiperativos e mais propensos a apresentar dificuldades de relacionamento com colegas da mesma idade cronológica, ou ainda com aqueles que se encontram nos mesmos níveis em déficit cognitivo (EINFELD; TONGE; FLORIO, 1997; GREER et al., 1997).

Kennedy, Kaye e Sadler (2006) relataram que $43 \%$ de uma amostra de 20 indivíduos com SWB preencheram critérios diagnósticos para transtorno de déficit de atenção/hiperatividade (TDAH). Leyfer et al. (2006) também mostraram que 65\% de 119 indivíduos com SWB foram diagnosticados com TDAH, assim como $54 \%$ para fobia específica.

Segundo Freeman et al. (2010), indivíduos com SWB e seus familiares têm consciência sobre as dificuldades em relação aos sintomas emocionais e de hiperatividade. Porém, quando se trata de problemas de relacionamento com colegas e comportamento pró-social, apresentam uma dificuldade de percepção quanto a estes déficits. Além do mais, Gosh e Pankau $(1994,1997)$ sugerem que, apesar de a SWB caracterizar-se por um comportamento altamente sociável, estes indivíduos mostram sérios problemas de adaptação social, que incluem dificuldades em desenvolver e se relacionar com colegas.

A qualidade de vida é um conceito de alcance abrangente, afetada de forma complexa pela saúde física, estado psicológico, nível de independência, relaçôes sociais e características do meio ambiente em que vive o indivíduo (WORLD..., 1993). Assim, apesar de, nesta pesquisa, os resultados obtidos sugerirem que os adolescentes com SWB apresentam uma qualidade de vida classificada, em sua opinião subjetiva, como 'boa', mostra-se um tanto quanto contraditório dizer que as questôes de saúde mental constatadas neste estudo não exercem qualquer influência.

Todavia, um estudo mostrou que crianças e adolescentes diagnosticadas com TDAH apresentaram prejuízos significativos em vários domínios associados à qualidade de vida, pois este diagnóstico está associado com problemas emocionais e comportamentais e, consequentemente, estes problemas repercutiram em seus pais, por lhes causarem sofrimento emocional e interferirem em atividades familiares (KLASSEN; MILLER; FINE, 2006). Outro estudo aponta que crianças com TDAH apresentam dificuldades em gerar respostas apropriadas (adaptativas) em casa, na escola e na comunidade (DUNN; BENNETT, 2012) por diferirem significativamente de crianças típicas em relação às respostas sensoriais (DUNN; BENNETT, 2012; SHIMIZU; BUENO; MIRANDA, 2014). John e Mervis (2010) demonstraram que a maioria das crianças com SWB que apresentaram pobre funcionamento adaptativo e executivo apresentaram maiores dificuldades de modulação sensorial e problemas de comportamentos. Inclusive, Amaral et al. (2013) observaram que indivíduos com SWB em idade escolar destacam-se pela personalidade amigável e boa narrativa, entretanto estas crianças mostram elevado índice de estresse; e que o estresse normalmente é consequência de uma personalidade otimista inadequada, pois esta proporciona maiores consequências desagradáveis causando o estresse, já que, normalmente, lidam de maneira inadequada com certas circunstâncias sociais.

Entretanto, ao considerar também a definição de qualidade de vida do World Health Organization (THE WHOQOL..., 1995) como

uma percepção individual da posição do indivíduo na vida, no contexto de sua cultura e sistema de valores nos quais ele está inserido e, em relação aos seus objetivos, expectativas, padróes e preocupaçôes,

sugere-se que os resultados deste estudo sejam válidos, apesar de o grupo com SWB, possivelmente, apresentar uma falha na compreensão sobre suas limitaçóes quanto às relações sociais, como foi apontado por Freeman et al. (2010); ou ainda, quanto à distorção da compreensão das dificuldades em manter relações sociais, e que a não percepção real da vida, provavelmente, se dá porque estes indivíduos possuem deficiência intelectual (MARTENS; WILSON; REUTENS, 2008; MERVIS et al., 2000).

O teste AUQEI se propóe a qualificar a qualidade de vida por intermédio da subjetividade do indivíduo avaliado, assim, os resultados desta pesquisa sáo válidos, já que os adolescentes com SWB conseguiram responder ao questionário. Desta forma, sugere-se que não se pode predizer que incapacidades ou déficits sempre interferiráo na qualidade de vida, assim como os possíveis sintomas clínicos não foram suficientes para mostrar que o bem-estar destes adolescentes estaria comprometido.

Ademais, o AUQEI é amplamente utilizado em investigaçóes que buscam avaliar a qualidade de vida 
em crianças saudáveis e com algum transtorno, como em um estudo que mostrou índices de qualidade de vida semelhantes em 20 crianças de 4 a 12 anos com transtorno do espectro autista, comparadas a 20 crianças normais, pareadas por gênero e idade (ELIAS; ASSUMPÇĀO JUNIOR, 2006).

O último ponto a ser discutido é o fato de que a intervenção em terapia ocupacional pode contribuir com o desenvolvimento dos indivíduos com SWB, já que eles apresentam dificuldades em diversas áreas que fazem parte do escopo desta profissão, tais como: raciocínio não verbal, percepção visuoespacial, representação espacial, memória de trabalho, planejamento motor e funçôes executivas (NUNES et al., 2013). Em seu estudo, Santos et al. (2015) observou que a intervenção com foco na motricidade, permite avanços positivos nas áreas da motricidade fina, equilíbrio e organização espacial nos indivíduos com SWB.

Assim, em acordo com os objetivos da intervenção e demanda de cada sujeito, diferentes abordagens e recursos podem ser utilizados por terapeutas ocupacionais a fim de melhorar a funcionalidade e o desempenho desses indivíduos em seu cotidiano. Dentre algumas técnicas de intervenção, a terapia de Integração Sensorial desenvolvida pela terapeuta ocupacional e neurocientista Jean Ayres (1972) pode ser uma das opçóes, já que visa ter como resultado uma resposta adaptativa aos estímulos vivenciados pelo indivíduo (SHIMIZU; MIRANDA, 2012). Entretanto, ainda é preciso maiores estudos para verificar a sua eficácia e efetividade nesta população.

A primeira limitação deste estudo se deu pelo fato de o tamanho da amostra ser dado por conveniência, afinal, foram avaliados todos os adolescentes cadastrados na ABSW que preenchiam os critérios de inclusão e que concordaram em participar desta pesquisa. Porém, limitou-se a amostra para adolescentes que residiam no Estado de São Paulo, portanto, necessário seria ampliar o tamanho da amostra e aplicar as avaliaçôes em outras regiōes brasileiras. A segunda limitação foi de não haver a correlação de que outros desfechos possam interferir na qualidade de vida destes adolescentes, como o comportamento adaptativo. Por fim, é escassa a literatura que descreve avaliaçóes ou intervenções da terapia ocupacional na SWB, o que muito dificulta a elaboração da discussão neste escopo.

\section{Conclusão}

Portanto, verifica-se que os adolescentes com SWB, em sua percepçáo de mundo, encontram-se satisfeitos com o tipo de qualidade de vida que levam em todos os segmentos da sociedade, independentemente de apresentarem sintomas clínicos que poderiam interferir de maneira negativa nessa qualidade. Desta forma, sugere-se que os indivíduos com SWB, provavelmente, podem apresentar uma distorção da realidade para o fator positivo/otimista, possivelmente baseada em uma das características comportamentais da própria síndrome associada à deficiência intelectual.

Diante dos aspectos investigados, propóe-se aos profissionais da área de Terapia Ocupacional e demais membros da equipe multi/inter/transdisciplinar que utilizem a metodologia aplicada neste estudo para avaliar e enriquecer o trabalho com os indivíduos com SWB, já que eles compreenderam as questôes do protocolo de qualidade de vida e responderam às questóes de forma adequada. Visto que esta síndrome tem sido pouco abordada pela área da terapia ocupacional, novos estudos poderão ser realizados a fim de proporcionar maiores informaçóes, desenvolver diferentes metodologias, entre outras, que contribuam com todos os envolvidos, e que, consequentemente, possam melhorar e enriquecer os métodos e os objetivos de intervençốes na SWB e também colaborar com a literatura.

\section{Referências}

AMARAL, V. A. A. S. et al. Estresse em crianças e adolescentes com Síndrome de Williams-Beuren em idade escolar. Psicologia Escolar e Educacional, Maringá, v. 17, n.1, p. 105-113, 2013.

ASSOCIAÇĀO BRASILEIRA DE EMPRESAS EM PESQUISA - ABEP. Critério de classificação econômica Brasil. São Paulo: ABEP, 2011. Disponível em: <http:// www.abep.org/criterio-brasil>. Acesso em: 22 jan. 2011.

ASSUMPÇÃO, J. R. et al. Escala de avaliação de qualidade de vida: (AUQEI - Autoquestionnaire Qualité de Vie Enfant Imagé) validade e confiabilidade de uma escala para qualidade de vida em crianças de 4 a 12 anos. Arquivos de Neuro-Psiquiatria, São Paulo, v. 58, n. 1, p. 119-127, 2000.

AYRES, A. J. Sensory integration and the child. Los Angeles: Western Psychological Services, 1972.

BEADLE-BROWN, J. et al. Changes in skills for people with intellectual disability: a follow-up of the Camberwell Cohort. Journal of Intellectual Disability Research, Malden, v. 44, p. 12-24, 2000. Suplemento 1.

BEUREN, A. J.; APITZ, J.; HARMJANZ, D. Supravalvular aortic stenosis in association with mental retardation and certain appearance. Circulation, Boston, v. 26, p. 1235-1240, 1962.

CALMAN, K. C. Quality of life in cancer patients: an hypothesis. Journal of Medical Ethics, London, v. 10, n. 3, p. 124-127, 1984. 
DANTAS, E. H. M. Fatores afetivos indispensáveis para o sucesso nos programas de atividade física para a terceira idade. Revista Brasileira de Atividade Física e Saúde, Pelotas, v. 2, n. 2, p. 75-82, 1997.

DEL COLE, C. G. et al. Análise do comportamento e do desempenho em atividades básicas e instrumentais de vida diária em adolescentes com Síndrome de Williams-Beuren. Temas sobre Desenvolvimento, São Paulo, v. 19, n. 104, p. 42-46, 2013.

DUNN, W.; BENNETT, D. Patterns of sensory processing in with attention deficit hyperactivity disorder. OTJR: Occupation, Participation and Health, Thousand Oaks, v. 22, n. 1, p. 4-15, 2012.

DYKENS, E. M. Anxiety, fears, and phobias in persons with Williams syndrome. Developmental Neuropsychology, Philadelphia, v. 23, n. 1-2, p. 291-316, 2003.

EINFELD, S. L.; TONGE, B. J.; FLORIO, T. Behavioural and emotional disturbance in individuals with Williams syndrome. American Journal of Mental Retardation, Washington, v. 102, n. 1, p. 45-53, 1997.

EINFELD, S. L.; TONGE, B. J.; REES, V. W. Longitudinal course of behavioral and emotional problems in Williams syndrome. American Journal on Mental Retardation, Washington, v. 106, n. 1, p. 73-81, 2001.

ELIAS, A. V.; ASSUMPÇÃO JÚNIOR, F. B. Quality of life and autism. Arquivos de Neuro-Psiquiatria, São Paulo, v. 64, n. 2a, p. 295-299, 2006.

EWART, A. K. et al. Hemizygosity at the elastin locus in a developmental disorder Williams syndrome. Nature Genetics, New York, v. 5, n. 1, p. 11-16, 1993.

FIGUEIREDO, V. L. M. et al. Propriedades psicométricas dos itens do teste WISC-III. Psicologia em Estudo, Maringá, v. 13, n. 3, p. 585-592, 2008.

FLEITLICH-BILYK, B.; GOODMAN, R. The prevalence of child psychiatric disorders in Southeast Brazil. Journal of The American Academy of Child \& Adolescent Psychiatry, Washington, v. 43, n. 6, p. 727-734, 2004.

FREEMAN, K. et al. Williams Syndrome: the extent of agreement between parent and self report of psychological difficulties. European Psychiatry, Strasbourg, v. 24, n. 3, p. 167-175, 2010.

FU, T. J. Profile and development of adaptive behavior in adolescents and adults with Williams Syndrome. International Journal of Information and Education Technology, Singapura, v. 5, n. 12, p. 931-935, 2015.

GOSH, A.; PANKAU, R. Social-emotional and behavioural adjustment in children with Williams syndrome. American Journal of Medical Genetics, Malden, v. 53, n. 4, p. 335-339, 1994.

GOSH, A.; PANKAU, R. Personality characteristics and behavior problems in individuals of different ages with Williams syndrome. Developmental Medicine and Child Neurology, Malden, v. 39, n. 8, p. 527-533, 1997.
GREER, M. K. et al. Cognitive, adaptive, and behavioral characteristics of Williams syndrome. American Journal of Medical Genetics, Malden, v. 74, n. 5, p. 521-525, 1997.

HAAS, B. W.; REISS, A. L. Social brain development in Willians syndrome: the current status and directions for future research. Frontiers in Psychology, Lausanne, v. 3, n. 186, p. 1-13, 2012.

HILLIER, L. W. et al. The DNA sequence of human chromosome 7. Nature, New York, v. 424, n. 6945, p. 157-164, 2003

HINDS, P. Quality of life in children and adolescents with cancer. Seminars in Oncology Nursing, New York, v. 6, n. 4, p. 285-291, 1990.

JANSEN, K. et al. Transtornos mentais comuns e qualidade de vida em jovens: uma amostra populacional de Pelotas. Cadernos de Saúde Pública, Rio de Janeiro, v. 27, n. 3, p. 440-448, 2011.

JÄRVINEN-PASLEY, A. et al. Defining the social phenotype in Williams syndrome: a model for linking gene, brain, and cognition. Development and Psychopathology, Cambridge, v. 20, n. 1, p. 1-35, 2008.

JAVINEN, A. M.; BELLUGI, U. What does Williams syndrome reveal about the determinants of social behavior? Frontiers in Human Neuroscience, Lausanne, v. 7, n. 321, p. 1-6, 2013.

JOHN, A. E.; MERVIS, C. B. Sensory modulation impairments in children with Williams Syndrome. American Journal of Medical Genetics Part C: Seminars in Medical Genetics, Malden, v. 154, n. 2, p. 266-276, 2010.

KENNEDY, J. C.; KAYE, D. L.; SADLER, L. S. Psychiatric diagnoses in patients with Williams syndrome and their families. Jefferson Journal of Psychiatry, Philadelphia, v. 20, n. 1, p. 22-31, 2006.

KLASSEN, A. F.; MILlER, A.; FINE, S. Agreement between parent and child report of quality of life in children with attention-deficit/hyperactivity disorder. Child: Care, Health and Development, Malden, v. 32, n. 4, p. 397-406, 2006

KORENBERG, J. Down syndrome: a molecular understanding of the origin of phenotypes. In: EPSTEIN, C. The phenotypic mapping of Down syndrome and other aneuploid conditions. New York: Wiley-Liss, 1993. p. $87-$ 115.

LEYFER, O. T. et al. Prevalence of psychiatric disorders in 4-16-years-olds with Williams Syndrome. American Journal of Medical Genetics Part B: Neuropsychiatric Genetics, San Francisco, v. 141B, n. 6, p. 615-622, 2006.

MALONE, D. M.; STONEMAN, Z. Cognitive play of mentally retarded preschoolers: observations in the home and school. American Journal of Mental Retardation, Washington, v. 94, n. 5, p. 475-487, 1990.

MANIFICAT, S.; DAZORD, A. Évaluation de la qualité de vie de l'enfant: validation d'un questionnaire, 
premiers resultants. Neuropsychiatrie de l'enfance et de l'adolescence, Limoges, v. 45, n. 1, p. 106-114, 1997.

MARTENS, M. A.; WILSON, S. J.; REUTENS, D. C. Williams syndrome: a critical review of the cognitive, behavioral, and neuroanatomical phenotype. Journal of Child Psychology and Psychiatry, Malden, v. 49, n. 6, p. 576-608, 2008.

MELLO, C. B. et al. Versão abreviada do WISC-III: correlação entre QI estimado e QI total em crianças brasileiras. Psicologia: Teoria e Pesquisa, São Paulo, v. 27, n. 2, p. 149-55, 2011.

MERVIS, C. B. et al. The Williams syndrome cognitive profile. Brain and Cognition, Cambridge, v. 44, n. 3, p. 604-628, 2000.

MERVIS, C. B. et al. Children with 7q11.23 duplication syndrome: psychological characteristics. American Journal of Medical Genetics Part A, Malden, v. 167, n. 7, p. 1436-1450, 2015.

NUNES, M. M. Avaliação do funcionamento cognitivo de pacientes com Sindrome de Williams-Beuren. 2010. 102 f. Tese (Doutorado em Ciências) - Universidade de São Paulo, São Paulo, 2010.

NUNES, M. M. et al. Assessment of intellectual and visuo-spatial abilities in children and adults with Williams Syndrome. Universitas Psychologica, Bogotá, v. 12, n. 2, p. 581-589, 2013.

SANTOS, A. P. M. et al. Efeitos da intervenção motora em uma criança com Síndrome de Williams. Revista Brasileira de Educação Especial, Marília, v. 21, n. 3, p. 423-432, 2015.

SAUR, A. M.; LOUREIRO, S. R. Qualidades psicométricas do questionário de capacidades e dificuldades: revisão da literatura. Estudos de Psicologia, Campinas, v. 29, n. 4, p. 619-629, 2012

SHIMIZU, V. T.; BUENO, O. F. A.; MIRANDA, M. C. Sensory processing abilities of children with ADHD. Brazilian Journal of Physical Therapy, São Carlos, v. 18, n. 4, p. 343-352, 2014.

SHIMIZU, V. T.; MIRANDA, M. C. Processamento sensorial na criança com TDAH: uma revisão da literatura. Revista Psicopedagogia, São Paulo, v. 29, n. 89, p. 256-268, 2012

SHIN, D.; JOHNSON, D. Avowed happiness as the overall assessment of the quality of life. Social Indicators Research, West Lafayette, v. 5, n. 1, p. 475-492, 1978.

SPARROW, S. S.; CICCHETTI, D. V.; BALLA, D. V. Interview edition survey form manual: vineland adaptive behavior scales. Circle Pines: American Guidance Service, 1984.

THE WHOQOL GROUP. The World Health Organization quality of life assessment (WHOQOL): position paper from the World Health Organization. Social Science and Medicine, London, v. 41, n. 10, p. 1403-1409, 1995.

WECHSLER, D. Manual for the Wechsler Intelligence Scale for children. San Antonio: Psychological Corporation, 1991.

WILLIAMS, J. C.; BARRATT-BOYES, B. G.; LOWE, J. B. Supravalvular aortic stenosis. Circulation, Dallas, v. 24, n. 6, p. 1311-1318, 1961.

WORLD HEALTH ORGANIZATION. WHOQOL study protocol: WHO (MNH7PSF/93.9). Geneva, 1993. Disponível em: <http://www.who.int/mental_health/ media/68.pdf>. Acesso em: 11 jun. 2011.

\section{Contribuição dos Autores}

Luísa realizou as avaliaçôes citadas neste estudo, suas análises e assistiu na concepção do texto. Andrea orientou o projeto de pesquisa financiado pela FAPESP e realizou a revisão do artigo. Rosana Aparecida contribuiu com as análises e com a revisão do texto. Carolina supervisionou as avaliaçóes realizadas por Luísa e assistiu nas análises, na redação e na revisão do texto. Todas as autoras aprovaram a versão final do texto.

\section{Fonte de Financiamento}

FAPESP: processo no 2011/19421-0. 